\title{
TÉCNICA PARA IDENTIFICAÇÃO DE OVOS DE Dioctophyme renale EM URINA DE GATOS, EMPREGADA M SÍLICA
}

\author{
Alexsander Ferraz ${ }^{1}$ \\ Bruna dos Santos Pires ${ }^{2}$ \\ Tainá Ança Evaristo ${ }^{3}$ \\ Soliane Carra Perera ${ }^{4}$ \\ Leandro Quintana Nizoli ${ }^{5}$ \\ Josaine Cristina da Silva Rapetti ${ }^{5}$
}

\begin{abstract}
RESUMO
A dioctofimatose é uma doença parasitária causada pelo helminto Dioctophyme renale, tendo como hospedeiros definitivos, cães, gatos, animais silvestres e também o homem, pois este parasito possui potencial zoonótico. Acomete os rins dos seus hospedeiros definitivos, geralmente o direito, mas podem ser encontrados também em outros locais. Embora seja descrito maior ocorrência de casos em cães, também há relatos desta parasitose em gatos. Uma das formas de diagnóstico é através da visualização de ovos do $D$. renale através do exame de urina. Porém, pelas características comportamentais dos gatos, a coleta de urina nesta espécie é de difícil execução. Portanto, através do estudo de técnicas para pesquisa de ovos deste parasito na urina dos gatos em sílica, presente nas caixas de areia, procura-se facilitar o diagnóstico nestes animais. Para realização deste trabalho, amostras de sílica foram contaminadas com urina contendo ovos de $D$. renale. As técnicas utilizadas para pesquisa de ovos foram: centrífugo-flutuação em solução hipersaturada glicosada, em duas densidades: $1.230 \mathrm{~g} / \mathrm{ml}$ e $1.275 \mathrm{~g} / \mathrm{ml}$; flutuação espontânea em solução hipersaturada glicosada, em duas densidades: $1,230 \mathrm{~g} / \mathrm{ml}$ e $1,275 \mathrm{~g} / \mathrm{ml}$ e centrífugo-sedimentação. A avaliação das amostras foi realizada em diferentes tempos, distribuídos da seguinte forma: momento zero, 6 horas, 12 horas, 18 horas, 24 horas, 36 horas, 48 horas, 72 horas, 120 horas, 168 horas, 240 horas e 336 horas após contaminação do material. A técnica de centrífugo-sedimentação foi a que apresentou o melhor resultado, sendo possível identificar ovos morfologicamente viáveis de $D$. renale durante os 14 dias de análise.
\end{abstract}

Palavras-chave: parasito, rins, diagnóstico, felinos.

\section{TECHNIQUE FOR IDENTIFYING EGGS OF Dioctophyme renale IN CAT URINE, EMPLOYED IN SILICA}

\begin{abstract}
Dioctofimatosis is a parasitic disease caused by the helminth Dioctophyme renale, having as definitive hosts, dogs, cats, wild animals and also man, as this parasite has zoonotic potential. It affects the kidneys of its definitive hosts, usually the right, but can also be found in other places. Although a higher occurrence of cases in dogs has been described, there are also reports of this parasitosis in cats. One of the ways of diagnosing the disease is through the visualization

\footnotetext{
${ }^{1}$ Médico Veterinário, Especialista em Doenças e Zoonoses Parasitárias, Doutorando do Programa de Pós Graduação em Veterinária da UFPel. - Universidade Federal de Pelotas.xanderferraz@yahoo.com.br

2 Médica Veterinária, Residente Multiprofissional na área de Clinica Cirurgica de Animais de Companhia na Universidade Federal de Pelotas

${ }^{3}$ Graduanda do curso de Medicina Veterinária da Universidade Federal de Pelotas

${ }^{4}$ Médica Veterinária, Doutora pelo Programa de Pós Graduação em Veterinária da UFPel

${ }^{5}$ Docente do curso de Medicina Veterinária da Universidade Federal de Pelotas.
} 
of $D$. renale eggs through the urine exam. However, due to the behavioral characteristics of cats, urine collection in this species is difficult to perform. Therefore, through the study of techniques to search for eggs of this parasite in the urine of cats in silica, present in litter boxes, we seek to facilitate the diagnosis in these animals. To carry out this work, silica samples were contaminated with urine containing $D$. renale eggs. The techniques used for egg research were: centrifugal-flotation in glycosated hypersaturated solution, in two densities: $1,230 \mathrm{~g} / \mathrm{ml}$ and $1,275 \mathrm{~g} / \mathrm{ml}$; spontaneous fluctuation in glycosated hypersaturated solution, in two densities: $1.230 \mathrm{~g} / \mathrm{ml}$ and $1.275 \mathrm{~g} / \mathrm{ml}$ and centrifugal-sedimentation. The evaluation of the samples was carried out at different times, distributed as follows: time zero, 6 hours, 12 hours, 18 hours, 24 hours, 36 hours, 48 hours, 72 hours, 120 hours, 168 hours, 240 hours and 336 hours after contamination of the material. The centrifugal-sedimentation technique showed the best result, making it possible to identify morphologically viable eggs from $D$. renale during the 14 days of analysis.

Key words: parasite, kidneys, diagnosis, felines.

\section{TÉCNICA DE IDENTIFICACIÓN DE HUEVOS DE Dioctophyme renale EN ORINA DE GATO, UTILIZADA EN SÍLICE}

\section{RESUMEN}

La dioctofimatosis es una enfermedad parasitaria causada por el helminto Dioctophyme renale, que tiene como hospedadores definitivos, perros, gatos, animales salvajes y también el hombre, ya que este parásito tiene potencial zoonótico. Afecta los riñones de sus huéspedes definitivos, generalmente el derecho, pero también se puede encontrar en otros lugares. Aunque se ha descrito una mayor incidencia de casos en perros, también hay informes de esta parasitosis en gatos. Una de las formas de diagnóstico es a través de la visualización de los huevos de D. renale a través del examen de orina. Sin embargo, debido a las características de comportamiento de los gatos, la recolección de orina en esta especie es difícil de realizar. Por lo tanto, a través del estudio de técnicas para buscar huevos de este parásito en la orina de gatos en sílice, presentes en cajas de arena, buscamos facilitar el diagnóstico en estos animales. Para llevar a cabo este trabajo, las muestras de sílice se contaminaron con orina que contenía huevos de $D$. renale. Las técnicas utilizadas para investigar los huevos fueron: flotación centrífuga en solución hipersaturada glicosilada, en dos densidades: $1.230 \mathrm{~g} / \mathrm{ml}$ y $1.275 \mathrm{~g} / \mathrm{ml}$; fluctuación espontánea en solución glicosilada hipersaturada, en dos densidades: $1.230 \mathrm{~g} / \mathrm{ml}$ y $1.275 \mathrm{~g} / \mathrm{ml}$ y sedimentación centrífuga. La evaluación de las muestras se realizó en diferentes momentos, distribuidos de la siguiente manera: tiempo cero, 6 horas, 12 horas, 18 horas, 24 horas, 36 horas, 48 horas, 72 horas, 120 horas, 168 horas, 240 horas y 336 horas después de la contaminación del material. La técnica de sedimentación centrífuga mostró el mejor resultado, permitiendo identificar huevos morfológicamente viables de $D$. renale durante los 14 días de análisis.

Palabras clave: parásito, riñones, diagnóstico, gatos

\section{INTRODUÇÃO}

A dioctofimatose é causada pelo parasito Dioctophyme renale, pertencente à ordem Enoplida, classe Nematoda e família Dioctophymetidae. Os exemplares machos medem entre 15 e $45 \mathrm{~cm}$ de comprimento por $3 \mathrm{a} 4 \mathrm{~mm}$ de diâmetro, enquanto as fêmeas medem de 20 a 100 $\mathrm{cm}$ de comprimento por 5 a $12 \mathrm{~mm}$ de diâmetro, sendo considerado o maior nematelminto já descrito (1). Sua coloração após retirada possui tons de vermelho-sangue, podendo ser atribuída a hematofagia (2). 
Os hospedeiros definitivos, seja estes, cães, gatos, animais silvestres ou o homem, eliminam os ovos de $D$. renale na urina, contaminando o meio ambiente (3). O ciclo possui um período de incubação em meio aquático, onde os ovos são ingeridos pelo hospedeiro intermediário, o anelídeo oligoqueta, e se tornam desta forma infectantes (4). A infecção ocorre pela ingestão de anelídeos aquáticos, assim como carne crua ou mal cozida de peixes e rãs que possuem em sua musculatura formas larvais encistadas viáveis (5).

A dioctofimatose é considerada uma zoonose, contudo, os relatos em humanos são escassos. Em cães, já houve relatos de infecções em vário estados do Brasil, como Espírito Santo (6), Minas Gerais (7), Paraná (8), Rio Grande do Sul (9), Rio de Janeiro (10), São Paulo (11), entre outros.

Embora seja descrito maior ocorrência de casos de dioctofimatose em cães, alguns trabalhos relatam esta parasitose em gatos, como Pedrassani et al. (12) em Canoinhas/SC e Silva et al. (13), que descreveram o primeiro relato de caso em gato doméstico no Rio Grande do Sul. Rappeti et al. (9), realizando um inquérito de casos de dioctofimatose em cães e gatos na região de Pelotas/RS, encontraram 95 casos registrados, destes, três foram em gatos.

O diagnóstico da dioctofimatose é realizado pela visualização do parasito em necropsias e em procedimentos cirúrgicos (14), exames ultrassanográficos (15) e pela visualização de ovos do $D$. renale através do exame da urina (16).

As formas adultas do parasito, localizam-se geralmente no rim direito dos seus hospedeiros definitivos, onde promovem a destruição progressiva do parênquima, reduzindo-o a uma cápsula fibrosa sem funcionalidade (8). Na literatura existe relatos de formas adultas encontradas livres na cavidade abdominal, ureteres, bexiga, uretra, bolsa escrotal, tecido subcutâneo inguinal, útero, ovários, glândulas mamária, cavidade torácica e pericárdio, cavidade abdominal, estômago, fígado e músculo reto abdominal $(17,18)$.

Atualmente, não há nenhuma opção farmacológica antiparasitária eficaz para tratamento de $D$. renale. Sabe-se que os anti-helmínticos de excreção renal, como o tiabendazol, eliminam metabólitos com baixa união com a $\beta$-tubulina dos nematódeos, além da rápida excreção urinária, dificultando sua penetração na cutícula dos helmintos (19). Mesmo se o parasito perdesse sua viabilidade através do uso de anti-helmínticos, ele não seria facilmente removido do interior do tecido renal devido a sua espessura e ao seu tamanho (20). Portanto, a nefrectomia do rim acometido é o procedimento de eleição para o tratamento (21). No entanto, diante do acometimento renal bilateral, a nefrotomia deve ser o método de escolha. Ademais, a laparotomia exploratória é eleita na remoção de parasitos livres na cavidade abdominal (22).

Através do estudo de técnicas para pesquisa de ovos deste parasito na urina de gatos domésticos em sílica, procura-se facilitar o diagnóstico nestes animais, pois a coleta de urina nesta espécie, pelas características comportamentais dos mesmos, é de difícil execução.

\section{MATERIAL E MÉTODOS}

A urina utilizada neste trabalho foi coletada de animais parasitados por D. renale, sendo este procedimento executado no Hospital de Clinicas Veterinárias (HCV), da Universidade Federal de Pelotas (UFPel).

A amostra de urina, contendo os ovos do parasito, foi acondicionada em pote de coleta, armazenada em caixa de isopor isotérmica, com gelo reciclável e encaminhada para posterior análise no Laboratório de Doenças Parasitárias (LADOPAR), da Faculdade de Veterinária (FaVet/UFPel).

As técnicas utilizadas para pesquisa de ovos de D. renale foram: centrífugo-flutuação em solução hipersaturada glicosada, com duas densidades: $1,230 \mathrm{~g} / \mathrm{ml}$ e $1,275 \mathrm{~g} / \mathrm{ml}$; flutuação espontânea em solução hipersaturada glicosada, com duas densidades: 1,230g/ml e 1,275g/ml e centrífugo-sedimentação. 
Amostras contendo 5 gramas de silica foram distribuídas em potes plásticos, sendo adicionado em cada uma destas, $500 \mu \mathrm{l}(0,5 \mathrm{ml})$ de urina contendo ovos de $D$. renale. A urina adicionada foi proveniente da mesma amostra.

A avaliação das amostras para identificação dos ovos foi realizada em diferentes tempos, distribuídos da seguinte forma: momento zero, 6 horas, 12 horas, 18 horas, 24 horas, 36 horas, 48 horas, 72 horas, 120 horas, 168 horas, 240 horas e 336 horas após contaminação do material.

As amostras foram confeccionadas em triplicata para cada uma das técnicas e tempo, totalizando 180 amostras (36 para cada técnica). O resultado foi obtido a partir da média das três amostras.

A técnica de centrifugo-flutuação, consistiu na homogeneização de $5 \mathrm{~g}$ de sílica contendo $500 \mu \mathrm{l}$ de urina e $15 \mathrm{ml}$ de água destilada, este material foi tamizado para um copo plástico, sendo posteriormente transferido para um tubo de ensaio e centrifugado por cinco minutos. Os sedimentos foram ressuspendidos com solução hipersaturada de açúcar (densidade de 1,230 e 1,275). Para promover a flutuação dos ovos, completou-se o volume do tubo até a formação de um menisco, para a colocação de uma lamínula $18 \times 18$, ficando em repouso por 20 minutos. A leitura foi realizada em microscopia óptica sob objetiva de $100 \mathrm{X}$

A técnica de flutuação espontânea foi executada a partir da homogeneização de $5 \mathrm{~g}$ de sílica contendo $500 \mu \mathrm{l}$ de urina contaminada e $25 \mathrm{ml}$ de solução hipersaturada de açúcar (duas densidades diferentes), o material foi tamizado para um copo plástico e transferido para um tubo de ensaio até a formação de um menisco. Após este processo, colocou-se uma lamínula de vidro sobre a solução por 20 minutos, pois o princípio da técnica, consiste que os ovos do nematoide flutuam pela diferença de densidade. A análise foi realizada em microscopia óptica com aumento de 100x.

A centrifugo-sedimentação foi realizada primeiramente com a homogeneização de $5 \mathrm{~g}$ de sílica contendo $500 \mu \mathrm{l}$ de urina e $15 \mathrm{ml}$ de água destilada, tamização do material para um copo plástico e transferência para um tubo de ensaio e centrifugação por 5 minutos. Ao final do processo, pipetou-se o sedimento em lâmina, sobrepondo-se uma lamínula. A observação do material foi realizada em microscopia óptica com aumento de 100x.

Foi atribuído uma graduação para expressar o número de ovos encontrados, de acordo com a seguinte escala: + (1 a 10 ovos); ++ (11 a 20 ovos $) ;+++$ (acima de 20 ovos).

\section{RESULTADOS E DISCUSSÕES}

A técnica de centrífugo-sedimentação por concentrar grande quantidade de ovos apresentou o melhor resultado, sendo possível identificar ovos morfologicamente viáveis de $D$. renale durante os 14 dias de análise (Tabela 1 ).

O D. renale, como a maioria dos nematoides, apresenta ovos com densidade baixa, o que os permite flutuarem em relação a diferença de densidade das soluções hipersaturadas utilizadas no experimento. Por este motivo, a técnica de flutuação espontânea também apresentou resultado satisfatório, possibilitando a identificação de ovos de D. renale em todas as analises, porém com menor número de ovos quando comparado com a técnica de centrífugosedimentação.

Ovos de D. renale em microscopia são elípticos, com coloração acastanhada, com parede espessa, rugosa e com um tampão bipolar. Possuem de uma a duas células logo após serem eliminadas na urina, medindo de 73 a $83 \mu \mathrm{m}$ de comprimento por 45 a $47 \mu \mathrm{m}$ de largura (21). Estas características morfológicas foram observadas após a realização da técnica de centrífugosedimentação e flutuação em solução hipersaturada, mesmo após os quatorze dias da preparação do material (Figura 1). 
Tabela 1. Contagem de ovos de $D$. renale, realizada através de diferentes técnicas e observadas em diferentes tempos.

\begin{tabular}{cccccc}
\hline Hora & $\begin{array}{c}\text { Flutuação } \\
\text { espontânea } \\
(1,230)\end{array}$ & $\begin{array}{c}\text { Flutuação } \\
\text { espontânea } \\
(1.275)\end{array}$ & $\begin{array}{c}\text { Centrífugo } \\
\text { Flutuação } \\
(1.230)\end{array}$ & $\begin{array}{c}\text { Centrífugo } \\
\text { Flutuação } \\
(1.275)\end{array}$ & $\begin{array}{c}\text { Centrífugo } \\
\text { Sedimentação }\end{array}$ \\
\hline 0 & ++ & ++ & + & + & +++ \\
6 & + & + & + & + & +++ \\
12 & ++ & ++ & + & + & +++ \\
18 & ++ & + & + & + & +++ \\
24 & ++ & + & + & + & ++ \\
36 & ++ & + & - & + & ++ \\
48 & +++ & + & - & + & ++ \\
72 & + & + & + & + & ++ \\
120 & ++ & + & + & + & ++ \\
168 & ++ & + & + & + & ++ \\
240 & ++ & + & + & + & ++ \\
336 & ++ & + & + & + & + \\
\hline
\end{tabular}

+(1 a 10 ovos $) ;++(11$ a 20 ovos $) ;+++($ acima de 20 ovos $)$

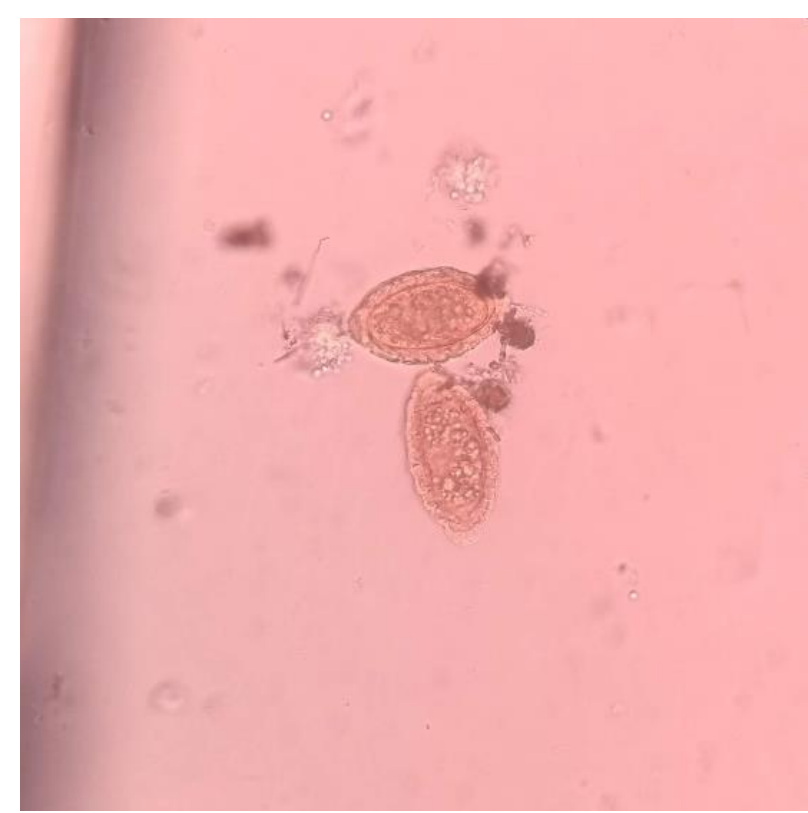

Figura 1. Ovos de D. renale, em amostra de sílica contendo urina, observados em microscopia ótica, com aumento de 400x. Fonte: Autores, 2019

Pedrassani et al. (20) relata que estudos relacionados com a morfologia dos ovos do parasito, a influência de temperatura para o desenvolvimento larval, a presença de formas larvais em hospedeiros paratênicos e a padronização de técnica para detecção de anticorpos para classe IgG, contra antígenos somáticos do parasito, em cães que albergam $D$. renale, se faz necessário para melhor conhecimento da dioctofimatose.

A elaboração de uma técnica laboratorial qualitativa para identificar ovos de $D$. renale em urina de gatos e que possa ser empregada em sílica, vem de acordo com o que cita Pedrassani et al. (20), possibilitando um novo leque de diagnóstico para presença de ovos do parasito em materiais utilizados em caixas de excretas de felinos.

Perera et al. (3), utilizando a técnica de Caldwell e Caldwell, analisaram amostras ambientais de areia, na região de uma clínica veterinária no município de Pelotas, onde muitos 
animais apresentavam diagnóstico positivo, e das 100 amostras testadas, 3\% continham ovos de D. renale.

Não existe na literatura, estudos com enfoque no diagnóstico a partir da observação de ovos de $D$. renale em caixas de areia de gatos, não sendo possível, desta forma, fazer uma análise comparativa com o presente estudo.

\section{CONCLUSÕES}

A partir deste estudo, conclui-se que a técnica de centrífugo-sedimentação obteve a maior efetividade para identificação de ovos de Dioctophyme renale, mesmo após o decorrer do tempo e da degradação ocorrente por fatores ambientais naturais nas amostras analisadas.

\section{REFERÊNCIAS}

1. Monteiro SG, Sallis ESV, Satainki DR. Infecção natural por trinta e quatro helmintos da espécie Dioctophyme renale (Goeze, 1782) em um cão. Rev FZVA [Internet]. 2002 [cited 2019 Apr 10];9(1):29-32. Available from: http://revistaseletronicas.pucrs.br/ojs/index.php/fzva/article/view/2149

2. Colpo CB, Silva AS, Monteiro SG. Occurrence of Dyoctophyma renale in dogs in the municipal disctrict of Uruguaiana - RS. Rev FZVA [Internet]. 2007 [cited 2019 Apr 10];14(2):175-8. Available from: http://revistaseletronicas.pucrs.br/ojs/index.php/fzva/article/view/2509

3. Perera SC, Capella GA, Pinto NB, Rappeti JCS, Muller G, Azambuja RHM, et al. First isolation of Dioctophyme renale eggs from an urban environment and identification of those from animal urine. Rev Bras Parasitol Vet [Internet]. 2017 [cited 2019 Apr 10];26(1):89-91. Available from: https://www.scielo.br/pdf/rbpv/v26n1/1984-2961-rbpv-S198429612016064.pdf

4. Alves GC, Silva DT, Neves MF. Dioctophyma renale: o parasita gigante do rim. Rev Cientif Eletronica Med Vet [Internet]. 2007 [cited 2019 Apr 10];4(8):1-6. Available from: http://www.faef.revista.inf.br/imagens_arquivos/arquivos_destaque/fPXZwNY3BuYYQ8 A_2013-5-24-11-38-31.pdf

5. Measures LN, Anderson RC. Centrarchid fish as paratenic hosts of the giant kidney worm, Dioctophyme renale (GOEZE, 1782), in Ontario, Canada. J Wildl Dis [Internet]. 1985 [cited 2019 Apr 10];21(1):11-9. Available from: https://www.jwildlifedis.org/doi/pdf/10.7589/0090-3558-21.1.11

6. Pereira BJ, Girardelli GL, Trivilin LO. Ocorrência de Dioctofimose em cães do município de Cachoeiro do Itapemirim, Espírito Santo, Brasil, no período de maio a dezembro de 2004. Rev Bras Parasitol Vet [Internet]. 2006 [cited 2019 Apr 10];15(3):123-5. Available from: https://www.redalyc.org/articulo.oa?id=397841459007

7. Poppi FP, Barcante JMP, Barcante TA. Primeiro relato de Dioctophyma renale parasitando o rim direito de um cão procedente do município de Poços de Caldas/ MG. Rev Bras Parasitol Vet. 2004;13 Supl 1:274. 
8. Leite LC. Lesões anato patológicas presentes na infecção por Dioctophyme renale (GOEZE, 1782) em cães domésticos (Canis familiaris, Linnaeus, 1758). Arch Vet Sci [Internet]. 2005 [cited 2019 May 26];10(1):95-101. Available from: https://revistas.ufpr.br/veterinary/article/view/4091/3318

9. Rappeti JCS, Mascarenhas CS, Perera SC, Muller G, Grecco FB, Silva LMC, et al. Dioctophyme renale (Nematoda: Enoplida) in domestic dogs and cats in the extreme south of Brazil. Rev Bras Parasitol Vet [Internet]. 2017 [cited 2019 May 26];26(1): 119-21. Available from: https://www.scielo.br/pdf/rbpv/v26n1/1984-2961-rbpv-S198429612016072.pdf

10. Marques LZ, Barros BAF, Gaudêncio FN, Rocha CNC, Batista LCSO. Dioctofimose em um cão proveniente do município de Valença, RJ: relato de caso. Acta Biomed Bras [Internet]. 2016 [cited 2019 Apr 10];7(2):116-20. Available from: https://www.actabiomedica.com.br/index.php/acta/article/view/149

11. Rocha MF, Padilha VS, Córdova RA, Marcondes MB. Dioctophyma renale em testículo de cão no município de curitibanos, SC, Brasil - relato de caso. Rev Cienc Vet Saude Publica [Internet]. 2018 [cited 2019 Apr 10];5(2):208-19. Available from: http://periodicos.uem.br/ojs/index.php/RevCiVet/article/view/41598/pdf

12. Pedrassani D, Wendt H, Rennau EA, Pereira ST, Wendt SBT. Dioctophyme renale Goeze, 1782 in a cat with a supernumerary kidney. Rev Bras Parasitol Vet [Internet]. 2013 [cited 2019 Apr 10];23(1):109-17. Available from: https://www.scielo.br/pdf/rbpv/v23n1/0103846x-rbpv-23-01-109.pdf

13. Silva CC, Cavalcanti GAO, Braga FVA, Guim TN, Gomes LGS, Rappeti JCS. Dioctophyme renale parasitism in domestic cat in Rio Grande do Sul, Brazil - first reported case. Semina Cienc Agrar [Internet]. 2017 [cited 2019 Apr 10];38(4):2215-20. Available from: http://www.uel.br/revistas/uel/index.php/semagrarias/article/view/25974

14. Sapin CF, Silva-Mariano LC, Piovesan AD, Fernandes CG, Rappeti JCS, Braga FVA, et al. Estudo anatomopatológico de rins parasitados por Dioctophyme renale em cães. Acta Sci Vet [Internet]. 2017 [cited 2019 May 26];45:1-7. Available from: http://www.ufrgs.br/actavet/45/PUB\%201441.pdf

15. Cottar BH, Dittrich G, Ferreira AA, Carvalho ACP, Albernaz VGP, Luz MT. Achados ultrassonográficos de cães parasitados por Dioctophyma renale: estudo retrospectivo. Vet Zootec [Internet]. 2012 [cited 2019 Apr 10];19(1 Supl 1):8-11. Available from: https://www.researchgate.net/publication/277150497_ACHADOS_ULTRASSONOGRA FICOS_DE_CAES_PARASITADOS_POR_Dioctophyma_renale__ESTUDO_RETROSPECTIVO

16. Li G, Liu C, Li F, Zhou M, Liu X, Niu Y. Fatal bilateral dioctophymatosis. J Parasitol. 2010;96(6):1152- 4.

17. Stainki DR, Pedrozo JCSR, Gaspar LFJ, Zanete RA, Silva AS, Monteiro SG. Urethral obstruction by Dioctophyma renale in puppy. Comp Clin Pathol. 2011;20(5):535-7. 
18. Caye P, Milech V, Lima CS, Braga FVA, Cleff MB, Rappeti JCS, et al. Intramuscular Dioctophyme renale surgically removed from dog - rare case report. Scholars J Agric Vet Sci [Internet]. 2018 [cited 2019 Apr 10];5(5):266-9. Available from: http://saspjournals.com/wp-content/uploads/2018/06/SJAVS-55-266-269-c.pdf

19. Lanusse CE. Farmacologia dos compostos anti-helmínticos. In: Charles TP. Controle de nematóides gastrintestinais em ruminantes. Juiz de Fora: Terezinha Padilha; 1996. p.1-44.

20. Pedrassani D, Hopper EGL, Avancini N. Morphology of eggs of Dioctophyme renale Goeze, 1782 (Nematoda: Dioctophymetidae) and influences of temperature on development of first-stage larvae in the eggs. Rev Bras Parasitol Vet [Internet]. 2009 [cited 2019 Apr 10];18(1):15-9. Available from: https://www.scielo.br/pdf/rbpv/v18n1/a03v18n1.pdf

21. Measures LN. Dioctophymatosis. In: Samuel WM, Pybus MJ, Kocan AA. Parasitic diseases of wild mammals. Ames: Iowa State University Press; 2001. p.357-64.

22. Birchard SJ, Sherding RG. Manual saunders: clínica de pequenos animais. 2a ed. São Paulo: Roca; 2003.

Recebido em: 17/07/2020 Aceito em: 05/10/2020 